\title{
The Nonperturbative Broken Phase Sphaleron Rate
}

\author{
Guy D. Moore ${ }^{\mathrm{a}}$

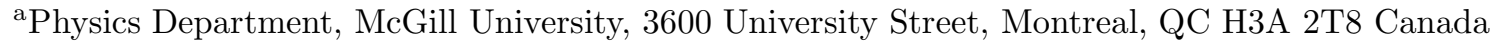

I present a technique for measuring the broken phase sphaleron rate nonperturbatively. There are three parts to the calculation: determination of the probability distribution of Chern-Simons number $N_{\mathrm{CS}}$; measurement of $\left\langle\left|d N_{\mathrm{CS}} / d t\right|\right\rangle_{N_{\mathrm{CS}}=1 / 2}$, the mean rate of change of $N_{\mathrm{CS}}$ at the barrier; and measurement of the "dynamical prefactor," the fraction of barrier crossings which result in a permanent integer change in $N_{\mathrm{CS}}$.

\section{Introduction}

The "sphaleron rate," the spacetime diffusion constant for Chern-Simons number $N_{\mathrm{CS}}$, is a key quantity in the study of electroweak baryogenesis, setting the efficiency of baryon number violation. It is also a very appropriate topic at this conference, since it is nothing but the topological susceptibility of the electroweak plasma, only in Minkowski, time;

$\Gamma \equiv \lim _{t \rightarrow \infty} \frac{\left\langle\left(N_{\mathrm{CS}}(t)-N_{\mathrm{CS}}(0)\right)^{2}\right\rangle}{V t}$.

$\Gamma$ can be determined reliably by perturbation theory in any regime where $g \phi(T) / T \gg \alpha_{w}$, where $\phi(T)$ is the Higgs condensate. However, this is precisely the regime where it is so small as to be of no physical consequence. We really want to know it in the symmetric phase at $T_{c}$ the phase transition temperature, to determine how many baryons can be produced; and in the broken phase at and immediately below $T_{c}$ to determine whether they are subsequently erased or are preserved until the present epoch.

Recently there has been significant progress in the symmetric phase rate. It has been shown analytically |1] and verified numerically [2] that "hard thermal loop" radiative corrections are important, and the rate is known with reasonable accuracy, though it has recently been demonstrated that there are logarithmic corrections [3] which are not well under control.

But the real time method cannot measure the broken phase rate; the rate is so low that no events will occur in any reasonable amount of time. Since we cannot extend the perturbative calculation beyond 1 loop, we will instead go about a nonperturbative measurement. We can use the dimensional reduction approximation and we will relentlessly; we also take the dynamics to be classical, regulated to give a physically reasonable cutoff to the hard thermal loops. For a much more detailed discussion of the work presented here, see [4].

\section{Defining and measuring $N_{\mathrm{CS}}$}

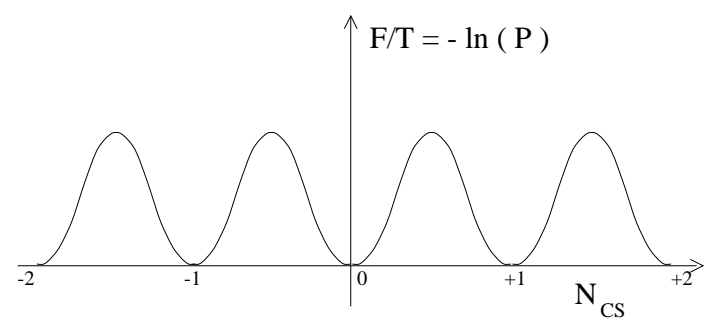

Figure 1. Cartoon of the free energy dependence on $N_{\mathrm{CS}}$

Here is the cartoon picture of why baryon number violation is slow in the broken phase. Because the Higgs condensate makes being half way between winding numbers energetically expensive, there is a free energy barrier to being at $N_{\text {CS }}=1 / 2$. Our method of determining the rate is to find the probability distribution of $N_{\mathrm{CS}}$, and to multiply the chance to be in a narrow range about $N_{\mathrm{CS}}=1 / 2$ by the mean crossing time of that range, set by $\left\langle\left|d N_{\mathrm{CS}} / d t\right|\right\rangle$. Then we need to determine what fraction of crossings are permanent. 
In the continuum, the meaning of $N_{\mathrm{CS}}$, modulo 1 , is the integral of $F \tilde{F}$,

$N_{\mathrm{CS}} \equiv \int d \tau\left(\frac{g^{2}}{8 \pi^{2}} \int d^{3} x B_{i}^{a}\left[D_{\tau}, A_{i}\right]^{a}\right)$.

Here $\tau$ parameterizes a path through the space of 3 -D configurations. The constant of integration is set by defining $N_{\mathrm{CS}}=0$ in vacuum. This is consistent since the above integral around any closed loop is the second Chern class of the loop, which is an integer. However, to make a nonperturbative study we need to find a lattice implementation for the above, which is touchy since no lattice definition of $F \tilde{F}$ is a total derivative. The best thing to do is to use the above definition for a unique specific path, the cooling path, defined by

$\frac{d U}{d \tau}=\left(D^{\alpha} U\right)\left(D^{\alpha} H_{\mathrm{YM}}\right)$,

with $H_{\mathrm{YM}}$ the Yang-Mills part of the 3-D Hamiltonian. This definition of $N_{\mathrm{CS}}$ is unique because the cooling path is; it is also minimally exposed to lattice problems since the cooling process very quickly eliminates UV fluctuations, which are responsible for any poor behavior in $F \tilde{F}$.

There are three problems we must take care of, though; a UV noise problem, a numerical cost problem, and the problem of turning a probability distribution for $N_{\mathrm{CS}}$ into a rate.

\section{The first two problems}

The first problem is that $N_{\mathrm{CS}}$ actually is not a very good measurable to be using. The problem is that it contains a lot of UV noise not related to topology. Our discussion will follow that of Ambjørn and Krasnitz [5]. In the UV the 3-D classical thermal theory we are studying behaves at leading order like 3 copies of the abelian theory, in which

$N_{\mathrm{CS}}=\frac{g^{2}}{32 \pi^{2}} \int d^{3} x \epsilon_{i j k} F_{i j}^{a} A_{k}^{a}$.

The mean square value at leading order in perturbation theory is then

$\left\langle N_{\mathrm{CS}}^{2}\right\rangle=\frac{g^{4} T^{2} V}{64 \pi^{4}} \int \frac{d^{3} p}{(2 \pi)^{3}} \frac{1}{p^{2}}$, which is linearly UV divergent. The size of the divergence is extensive in volume and will be cut off by the lattice spacing, leading to a $1 / a$ behavior.

The solution is not to measure $N_{\mathrm{CS}}$ itself, but $N_{\text {CS }}$ after some preliminary cooling has been performed;

$N \equiv \int_{\tau_{0}}^{\infty} d \tau\left(\frac{g^{2}}{8 \pi^{2}} \int d^{3} x B_{i}^{a}\left[D_{\tau}, A_{i}\right]^{a}\right)$.

To illustrate that this does the trick, we show $N_{\text {CS }}$ and $N$ for a period of Hamiltonian evolution in the broken phase, and $N$ for a sphaleron transition:
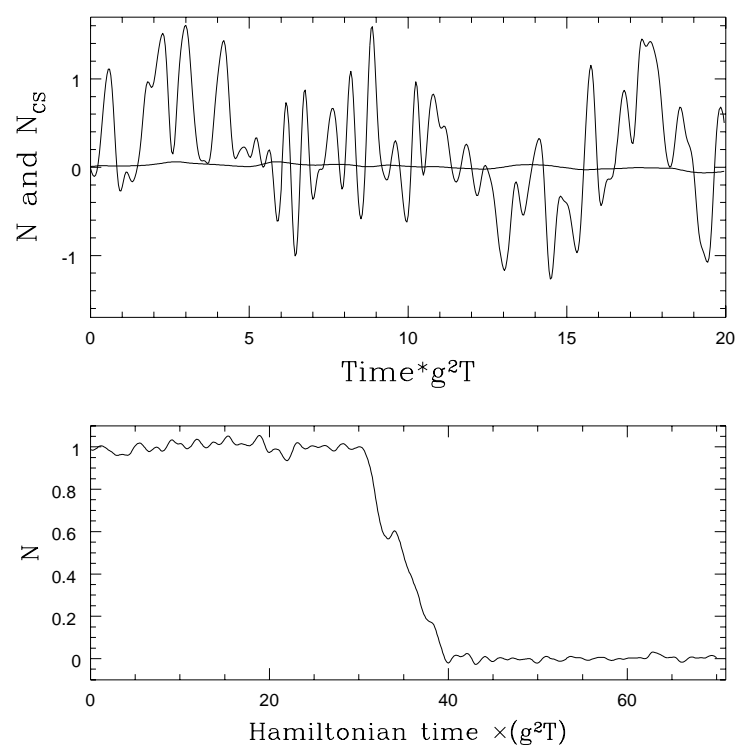

Figure 2. Above: $N_{\mathrm{CS}}$ (oscillating like mad) and $N$ (always near zero) for a Hamiltonian trajectory in the broken phase. Below: $N$ during a sphaleron.

This graphically illustrates that using $N$ cuts out irrelevant UV noise, while still capturing interesting topological information.

There is still a problem with using $N$, the numerical cost involved in performing the cooling, which we must do thousands of times to get an accurate Monte-Carlo study of the probability distribution. We finesse this problem by observing that, since cooling eliminates the UV excitations, it quickly renders the lattice fields smooth 
enough that we lose no information by blocking by a factor of 2 . When we use a classically improved Hamiltonian and definition of $F \tilde{F}$ after the blocking, there is almost no difference between the blocked and unblocked measurement of $N$, provided we cool by at least $\tau>1.25 a^{2}$ before blocking. For lattices larger than about $28^{3}$ we can double block.

\section{Turning a probability into a rate}

We can now find the ( $\tau_{0}$ dependent) probability distribution of $N$ by conventional multicanonical means. To turn this into a rate, we first measure $\langle|d N / d t|\rangle$ (sampled over $N=1 / 2$ configurations) by making a canonically distributed sample of $N=1 / 2$ configurations, generating momenta from the canonical ensemble, and evolving for a very short Hamiltonian time, measuring $N$ before and after. The mean rate of $N=1 / 2$ crossings per 4 -volume is then

$\frac{\Gamma}{\text { prefactor }}=\frac{P(|N-0.5|<\epsilon)}{2 \epsilon} \times \frac{\langle|d N / d t|\rangle}{V}$.

To measure the dynamical prefactor, which is the fraction of crossings which lead to permanent integer change in $N_{\mathrm{CS}}$, we use the same $N=1 / 2$ sample but continue the Hamiltonian time evolution, both forward and backward in time, until the system settles about a vacuum; then we count how many crossings and how many permanent $N$ changes occur. The fraction of crossings leading to permanent $N$ change is

$\frac{1}{N_{\text {samp }}} \sum \frac{1}{\# \text { crossings }} \times \begin{cases}1 & N_{\text {fin }} \neq N_{\text {init }} \\ 0 & N_{\text {fin }}=N_{\text {init }}\end{cases}$

For very large Debye mass squared $m_{D}^{2}$ there are multiple crossings, but for realistic $m_{D}^{2}$ the prefactor is about 0.4 .

\section{Results}

The final results for $\Gamma$ are compared to perturbation theory (two loop effective potential but tree kinetic terms) in Figure 1 . Baryon number erasure after the phase transition is prevented if $\Gamma<10^{-7} \alpha_{W}^{4} T^{4}$, which occurs if $x<0.037$.

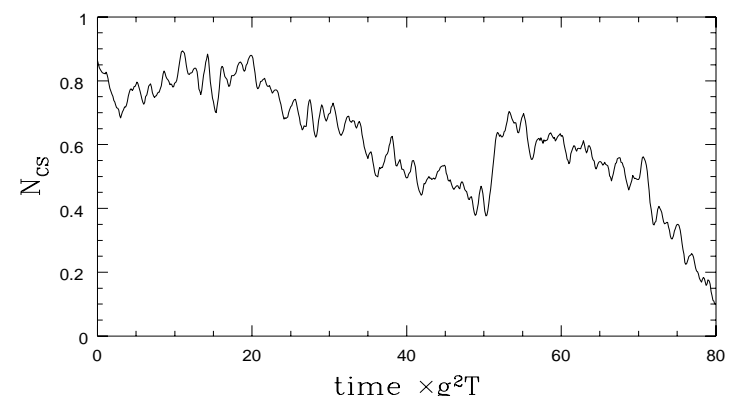

Figure 3. Multiple crossings when $m_{D}^{2}=43 g^{4} T^{2}$, a very large hard thermal loop strength.

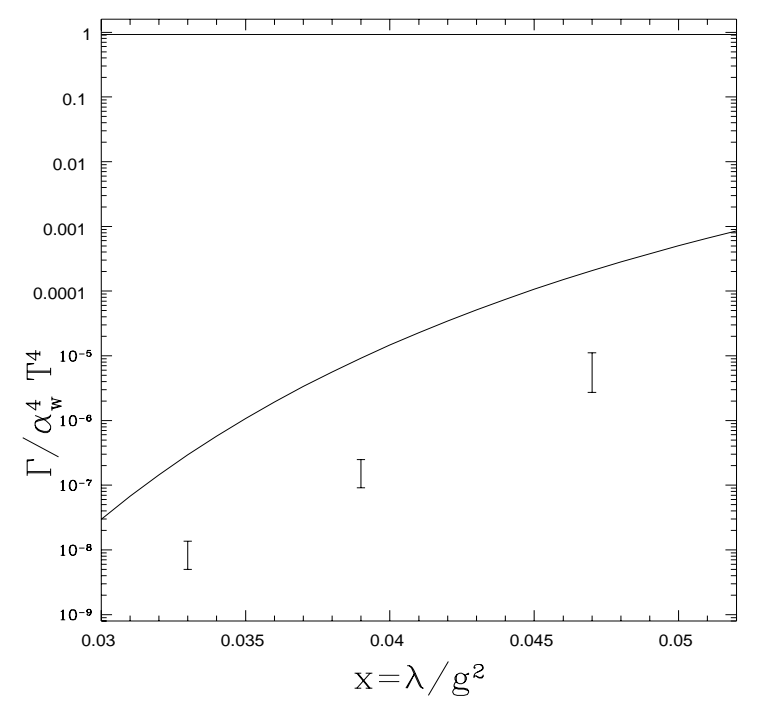

Figure 4. Nonperturbative value of $\Gamma\left(T_{c}\right)$ (points with bars) versus perturbative estimate (curve) and the symmetric phase rate (curve at top), as a function of $x \equiv \lambda / g^{2}$.

\section{REFERENCES}

1. P. Arnold, D. Son, and L. Yaffe, Phys. Rev. D 55, 6264 (1997).

2. G. D. Moore, C. Hu, and B. Müller, Phys. Rev. . D 58, 045001 (1998).

3. D. Bödeker, Phys. Lett. B 426, 351 (1998).

4. G. D. Moore, hep-ph/9805264.

5. J. Ambjørn and A. Krasnitz, Phys. Lett. B 362, 97 (1995). 